\title{
FEEDBACK STABILIZATION OF SEMILINEAR HEAT EQUATIONS
}

\author{
V. BARBU AND G. WANG
}

Received 24 October 2002

This paper is concerned with the internal and boundary stabilization of the steady-state solutions to quasilinear heat equations via internal linear feedback controllers provided by an LQ control problem associated with the linearized equation.

\section{Introduction}

Let $\Omega \in \mathbb{R}^{n}$ be an open and bounded subset with smooth boundary $\partial \Omega$, let $\omega \subset$ $\Omega$ be an open subset, and let $m$ be the characteristic function of $\omega$. Let $Q=\Omega \times$ $(0, \infty)$ and $\Gamma=\partial \Omega \times(0, \infty)$. We will study here the internal feedback stabilization of steady-state solutions to the quasilinear heat equation

$$
\begin{gathered}
y_{t}(x, t)-\Delta y(x, t)+f(x, y(x, t), \nabla y(x, t))=m(x) u(x, t) \text { in } Q, \\
y(x, 0)=y_{0}(x) \quad \text { in } \Omega, \\
y(x, t)=0 \quad \text { on } \Gamma,
\end{gathered}
$$

and the boundary feedback stabilization to the semilinear heat equation

$$
\begin{gathered}
z_{t}(x, t)-\Delta z(x, t)+a(x) z(x, t)+g(z(x, t))=0 \quad \text { in } Q, \\
z(x, 0)=z_{0}(x) \quad \text { in } \Omega, \\
z(x, t)=u(x, t) \quad \text { on } \Gamma,
\end{gathered}
$$

where $a \in L^{\infty}(\Omega)$.

Let $y_{e}$ be a steady-state solution to (1.1), that is,

$$
\begin{gathered}
-\Delta y_{e}(x)+f\left(x, y_{e}(x), \nabla y_{e}(x)\right)=0 \quad \text { in } \Omega, \\
y_{e}(x)=0 \quad \text { on } \partial \Omega .
\end{gathered}
$$

Copyright (C) 2003 Hindawi Publishing Corporation Abstract and Applied Analysis 2003:12 (2003) 697-714 2000 Mathematics Subject Classification: 35BXX, 93B15, 76D05, 76D55, 35B40 URL: http://dx.doi.org/10.1155/S1085337503212100 
It turns out (see $[1,3])$ that if $f=f(x, r)$ is locally Lipschitz continuous in $r$, then any sufficiently smooth steady-state solution $y_{e}$ is locally controllable in any finite time $T$ and so, in particular, it is stabilizable.

In the present paper, we will prove (Theorem 2.1) that if $f$ is of polynomial growth in $(r, \theta)$ with suitable exponents (which will be precised later), then (1.1) is locally exponentially stabilizable by the feedback controller

$$
u=-m P\left(y-y_{e}\right)
$$

where $P$ is the solution to an algebraic Riccati equation associated with the linearized equation. We must stress that we do not use the local null controllability of (1.1) to get the stabilization but approach the stabilization in a different way which is inspired by Lyapunov stability theory for finite-dimensional systems. More precisely, by defining an appropriate infinite horizon LQ problem with unbounded cost functional, we find a linear selfadjoint and positive operator $P$, which is the solution to an algebraic Riccati equation associated with the LQ problem such that the feedback law (1.4) makes $y_{e}$ locally exponentially stable.

In a similar way, we obtain a boundary feedback controller which locally exponentially stabilizes (1.2). Previously, such a result was obtained (see [2]) for 3-dimensional Navier-Stokes equations.

Throughout this paper, we let $H=L^{2}(\Omega)$ with the norm $|\cdot|$ and $V=H_{0}^{1}(\Omega)$ with the norm $\|\cdot\|$. We denote by $|\cdot|_{s}$ the norm of $L^{s}(\Omega)$ and by $\|\cdot\|_{r}$ the norm of $H^{r}(\Omega)$. We use $(\cdot, \cdot)$ to denote the inner product in $H$ and the paring between $V$ and $V^{\prime}$ and between $H^{r}(\Omega)$ and $\left(H^{r}(\Omega)\right)^{\prime}$, respectively. Use $|\cdot|_{\omega}$ and $(\cdot, \cdot)_{\omega}$ to denote the norm and the inner product of $L^{2}(\omega)$, respectively.

This paper is organized as follows. In Section 2, we present assumptions and the main results of the paper (Theorems 2.1 and 2.2) while in Sections 3 and 4, we prove Theorems 2.1 and 2.2, respectively.

\section{Assumptions and the main results}

Throughout this paper, we will assume the following conditions:

$\left(\mathrm{H}_{1}\right)$ the steady-state solution $y_{e}$ is smooth enough such that $y_{e}, \nabla y_{e} \in$ $C(\bar{\Omega})^{n}$;

$\left(\mathrm{H}_{2}\right)$ the function $f(x, r, \theta)$, where $\theta=\left(\theta_{1}, \ldots, \theta_{n}\right) \in \mathbb{R}^{n}$, is continuously differentiable with respect to all arguments, $f_{r}(x, \cdot, \theta)$ and $f_{\theta}(x, r, \cdot)$ are locally Lipschitz continuous, where $f_{\theta}=\left(f_{\theta_{1}}, \ldots, f_{\theta_{n}}\right)$. Moreover, $f$ satisfies the growth condition

$$
|f(x, r, \theta)| \leq C\left(\sum_{i=1}^{m}|r|^{p_{i}}+\sum_{j=1}^{l}|\theta|^{q_{j}}\right) \quad \forall x \in \Omega, r \in \mathbb{R}, \theta \in \mathbb{R}^{n}
$$


where $|\theta|^{2}=\sum_{i=1}^{n} \theta_{i}^{2}, m, l \geq 2$ are arbitrary positive integers, $p_{i}$ and $q_{j}$, $i=1, \ldots, m, j=1, \ldots, l$, are such that $1 \leq p_{1}<p_{2} \cdots<p_{m}, 1 \leq q_{1}<q_{2}<$ $\cdots<q_{l}$, and

(i) if $n=1$, then $p_{i}>1$ and $1<q_{j} \leq 2$ for all $i=2, \ldots, m$ and $j=2, \ldots, l$;

(ii) if $n=2$, then $1<p_{i} \leq 5$ and $1<q_{j} \leq 5 / 3$ for all $i=2, \ldots, m$ and $j=$ $2, \ldots, l$;

(iii) if $3 \leq n \leq 9$, then $1<p_{i} \leq(n+3) /(n-1)$ and $1<q_{j} \leq n /(n-1)$ for all $i=2, \ldots, m$ and $j=2, \ldots, l$;

(iv) if $n>9$, then $1<p_{i} \leq n /(n-3)$ and $1<q_{j} \leq n /(n-1)$ for all $i=$ $2, \ldots, m$ and $j=2, \ldots, l$;

$\left(\mathrm{H}_{3}\right)$ the function $g: R \rightarrow \mathbb{R}$ is continuous with $g(0)=0$ and satisfies the growth condition

$$
|g(y)| \leq C|y|^{p} \quad \forall y \in \mathbb{R}
$$

where $p$ satisfies

$$
1<p \leq \min \left\{2, \frac{n+1+4 \varepsilon}{n-1+4 \varepsilon}\right\}
$$

with some $\varepsilon>0$ arbitrary small but fixed.

The following notations will be used. We will omit all $x, t$ in the functions of $x$, $t$ if there is no ambiguity. Let $A=-\Delta$ with $D(A)=H^{2}(\Omega) \cap H_{0}^{1}(\Omega)$ and $A_{0} y=$ $f_{r}\left(x, y_{e}, \nabla y_{e}\right) y+f_{\theta}\left(x, y_{e}, \nabla y_{e}\right) \cdot \nabla y$. We denote by $A^{s}, s \in(0,1)$, the fractional power of operator $A$. Let $W=D\left(A^{1 / 4}\right)$ with the graph norm $|y|_{W}=\left|A^{1 / 4} y\right|$. Recall that (see [7, page 66] $D\left(A^{1 / 4}\right)=\left\{y \in H^{1 / 2}(\Omega) ;(\operatorname{dist}(x, \partial \Omega))^{-1 / 2} y \in L^{2}(\Omega)\right\}$.

Now we are ready to formulate the main results.

Theorem 2.1. Suppose that $\left(H_{1}\right)$ and $\left(H_{2}\right)$ hold. Then there exists a linear selfadjoint positive operator $P: H \rightarrow H$ with the domain $D(P)$ satisfying $V \subset D(P) \subset W$ such that the feedback controller

$$
u=-m P\left(y-y_{e}\right)
$$

exponentially stabilizes $y_{e}$ in a neighborhood $W_{\rho}=\left\{y_{0} \in W ;\left|y-y_{e}\right|_{W}<\rho\right\}$ of $y_{e}$. More precisely, for each $y_{0} \in W_{\rho}$, there is a solution $y \in C\left(\mathbb{R}^{+} ; H\right) \cap L_{\text {loc }}^{2}\left(\mathbb{R}^{+} ; V\right)$ to the closed-loop equation

$$
\begin{gathered}
y_{t}+A y+f(x, y, \nabla y)+m P\left(y-y_{e}\right)=0, \quad t>0, \\
y(0)=y_{0},
\end{gathered}
$$

such that

$$
\begin{gathered}
\int_{0}^{\infty}\left|A^{3 / 4} y(t)\right|^{2} d t \leq C\left|y_{0}-y_{e}\right|_{W}^{2}, \\
\left|y(t)-y_{e}\right| \leq C e^{-\gamma t}\left|y_{0}-y_{e}\right|_{W} \quad \forall t>0,
\end{gathered}
$$


where $\gamma>0$. Moreover, $P$ is the solution to the algebraic Riccati equation

$$
\left(\left(A+A_{0}\right) y, P y\right)+\frac{1}{2}|m P y|^{2}=\frac{1}{2}\left|A^{3 / 4} y\right|^{2} \quad \forall y \in D(A) .
$$

For the stabilization of (1.2), we will transform the boundary control problem into a distributed control problem via the method provided in [5]. We denote by $A$ again the extension of $A$ from $H$ to $(D(A))^{\prime}$ (the dual space of $D(A)$ ), that is, the operator defined by

$$
(A z, \varphi)=\int_{\Omega} z \Delta \varphi d x \quad \forall \varphi \in H_{0}^{1}(\Omega) \cap H^{2}(\Omega) .
$$

Let $A_{1} z=a z$ and $B: U \equiv L^{2}(\partial \Omega) \rightarrow(D(A))^{\prime}$ be defined by

$$
B u=A \theta,
$$

where $\theta$ is the solution to

$$
\Delta \theta=0 \quad \text { in } \Omega, \quad \theta=u \quad \text { on } \partial \Omega .
$$

We will denote by $|\cdot|_{U}$ and $(\cdot, \cdot)$ the norm and the inner product of $U$ and by $B^{*}$ the adjoint operator of $B$. It is well known (see $\left.[5,7]\right)$ that $\theta \in D\left(A^{1 / 4-\varepsilon}\right)$ for $\varepsilon>0$ arbitrarily small, and so, we have

$$
A^{-3 / 4-\varepsilon} B \in L(U, H)
$$

Then (1.2) can be written as (see [5])

$$
z^{\prime}+A z+A_{1} z+g(z)=B u, \quad t>0, \quad z(0)=z_{0} .
$$

Theorem 2.2. Suppose that hypothesis $\left(H_{3}\right)$ holds. Then there is a linear operator $P:\left(D\left(A^{1 / 4+\varepsilon}\right)\right)^{\prime} \rightarrow D\left(A^{1 / 4+\varepsilon}\right)$, where $\varepsilon>0$ is arbitrarily small but fixed, such that the operator $A+A_{1}+B B^{*} P$ generates a $C_{0}$-semigroup $S(t)$ on $\left(D\left(A^{1 / 4+\varepsilon}\right)\right)^{\prime}$ and the feedback law

$$
u=-B^{*} P y
$$

locally exponentially stabilizes (2.12). More precisely, there is a $\rho>0$ such that for any $z_{0} \in V_{\rho} \cap D\left(A^{1 / 4-\varepsilon}\right)$, where $V_{\rho}=\left\{\left(D\left(A^{1 / 4+\varepsilon}\right)\right)^{\prime} ;\left(P z_{0}, z_{0}\right)<\rho\right\}$, there is a mild solution $z \in C\left([0, \infty) ;\left(D\left(A^{1 / 4+\varepsilon}\right)\right)^{\prime}\right)$ to $(2.12)$ satisfying

$$
\begin{gathered}
\int_{0}^{\infty}\left|A^{1 / 4-\varepsilon} z(t)\right|^{2} d t \leq C\left|z_{0}\right|_{\left(D\left(A^{1 / 4+\varepsilon}\right)\right)^{\prime}}^{2}, \\
\left|A^{-1 / 4-\varepsilon} z(t)\right| \leq C e^{-\gamma t}\left|z_{0}\right|_{\left(D\left(A^{1 / 4+\varepsilon}\right)\right)^{\prime}}, \quad \forall t>0 .
\end{gathered}
$$




\section{Internal stabilization of quasilinear equation}

We consider the linearized equation

$$
\begin{gathered}
y_{t}+A y+A_{0} y=m u, \quad t>0, \\
y(0)=y^{0} \equiv y_{0}-y_{e},
\end{gathered}
$$

where $A$ and $A_{0}$ were given in Section 2, and the LQ optimal control problem

$$
\varphi\left(y^{0}\right)=\operatorname{Min}\left\{\frac{1}{2} \int_{0}^{\infty}\left(\left|A^{3 / 4} y(t)\right|^{2}+|u(t)|_{\omega}^{2}\right) d t, \text { subject to }(3.1)\right\} .
$$

We denote by $D(\varphi)$ the set of all $y^{0} \in H$ such that $\varphi\left(y^{0}\right)<\infty$. It is well known (see $[1,3])$ that, for each $y^{0} \in H$, the linear equation (3.1) is exactly null controllable on each interval $[0, T]$. More precisely, there is $u \in L^{2}\left(0, T ; L^{2}(\Omega)\right)$ and $t^{1 / 2} y \in$ $L^{2}(0, T ; D(A))$ satisfying (3.1) such that $y(T)=0$. If $y^{0} \in D\left(A^{1 / 4}\right)$, then $A^{3 / 4} y \in$ $L^{2}(0, T ; H)$, and so

$$
\varphi\left(y^{0}\right) \leq C\left|A^{1 / 4} y^{0}\right|^{2}, \quad \forall y^{0} \in D\left(A^{1 / 4}\right),
$$

because $\varphi$ is linear quadratic. (Here and throughout the paper, $C$ denotes several positive constants.)

On the other hand, we have

$$
\varphi\left(y^{0}\right) \geq C\left|A^{1 / 4} y^{0}\right|^{2} \quad \forall y^{0} \in D\left(A^{1 / 4}\right) .
$$

Indeed, it is easy to see that, for each $y^{0} \in D(\varphi)$, problem (3.2) has a unique solution $\left(y^{*}, u^{*}\right) \in L^{2}\left(\mathbb{R}^{+} ; D\left(A^{3 / 4}\right)\right) \times L^{2}\left(\mathbb{R}^{+} ; L^{2}(\omega)\right)$. Moreover, $y^{*} \in C\left(\mathbb{R}^{+} ; D\left(A^{1 / 4}\right)\right)$. If we multiply (3.1), where $y=y^{*}$ and $u=u^{*}$, by $A^{1 / 2} y^{*}$ and integrate it on $(0, \infty)$, we obtain

$$
\begin{aligned}
\frac{1}{2}\left|A^{1 / 4} y^{0}\right|^{2} & \leq \int_{0}^{\infty}\left(\left(A y^{*}, A^{1 / 2} y^{*}\right)+\left(A^{0} y^{*}, A^{1 / 2} y^{*}\right)+\left|u^{*}\right|_{\omega}\left|A^{1 / 2} y^{*}\right|\right) d t \\
& \leq C \int_{0}^{\infty}\left(\left|A^{3 / 4} y^{*}\right|^{2}+|u|_{\omega}^{2}\right) d t=C \varphi\left(y^{0}\right)
\end{aligned}
$$

because

$$
\begin{aligned}
\left|\left(A_{0} y^{*}, A^{1 / 2} y^{*}\right)\right| & \leq C\left(\left\|y_{e}\right\|_{C(\bar{\Omega})}+\left\|\nabla y_{e}\right\|_{C(\bar{\Omega})}\right)\left(\left|y^{*} \| A^{1 / 2} y^{*}\right|+\left|A^{1 / 2} y^{*}\right|^{2}\right) \\
& \leq C\left\|y^{*}\right\|^{2} .
\end{aligned}
$$

It follows immediately from (3.3) and (3.4) that $D(\varphi)=D\left(A^{1 / 4}\right) \equiv W$. Since the functional $\varphi$ is quadratic, there exists a linear selfadjoint positive operator 
702 Feedback stabilization of semilinear heat equations

$P: H \rightarrow H$ with domain $D(P) \subset W$ such that

$$
\frac{1}{2}\left(P y^{0}, y^{0}\right)=\varphi\left(y^{0}\right) \quad \forall y^{0} \in D(P) .
$$

Moreover, $P$ extends to all of $W$ and $P \in L\left(W, W^{\prime}\right)$.

Lemma 3.1. Let $\left(y^{*}, u^{*}\right) \in L^{2}\left(0, \infty ; D\left(A^{3 / 4}\right)\right) \times L^{2}\left(0, \infty ; L^{2}(\Omega)\right)$ be the optimal pair for problem (3.2) corresponding to $y^{0} \in D\left(A^{1 / 4}\right)$. Then

$$
u^{*}(t)=-m P y^{*}(t) \quad \forall t>0 .
$$

Moreover, $V \subset D(P)$, that is,

$$
|P y| \leq C\|y\| \quad \forall y \in V,
$$

and there exist $C_{i}>0, i=1,2$, such that

$$
C_{1}\|y\|_{W}^{2} \leq(P y, y) \leq C_{2}\|y\|_{W}^{2} \quad \forall y \in W .
$$

The operator $P$ is the solution to the algebraic Riccati equation

$$
\left(A y+A_{0} y, P y\right)+\frac{1}{2}|m P y|^{2}=\frac{1}{2}\left|A^{3 / 4} y\right|^{2} \quad \forall y \in D(A) .
$$

Proof. Estimate (3.10) follows immediately from (3.3) and (3.4). Since the quadratic cost functional (3.2) is unbounded on $H$, the conclusions of the lemma are not implied by the general theory of infinite-dimensional LQ control problems (see [5]). We treat it in the following way.

By the dynamic programming principle, it follows that for each $T>0,\left(y^{*}, u^{*}\right)$ is the solution to the optimal control problem

$$
\begin{aligned}
\operatorname{Min}\{ & \frac{1}{2} \int_{0}^{T}\left(\left|A^{3 / 4} y(t)\right|^{2}+|u(t)|_{\omega}^{2}\right) d t \\
& +\varphi(y(T)) ;(y, u) \text { subject to }(3.1)\} .
\end{aligned}
$$

By the maximum principle, we obtain that

$$
u^{*}(t)=m q^{T}(t) \quad \forall t \in[0, T),
$$

where $q^{T}$ is the solution to the adjoint equation

$$
\begin{gathered}
\frac{d}{d t} q^{T}-\left(A+A_{0}\right)^{*} q^{T}=A^{3 / 2} y^{*}, \quad t \in(0, T), \\
q^{T}(T)=-P y^{*}(T) .
\end{gathered}
$$

Since $q^{T} \in W^{\prime} \subset V^{\prime}$, it follows from the standard existence theory for linear evolution equations that $q^{T} \in L^{2}(0, T ; H) \cap C\left([0, T], V^{\prime}\right)$. Moreover, if $y^{0} \in V$, 
we have $q^{T} \in C([0, T] ; H)$. Indeed, $y^{*} \in L^{2}(0, T ; D(A))$ if $y^{0} \in V$. If we set $z=$ $A^{-1 / 2} q^{T}$, it follows from (3.4) that

$$
z^{\prime}-A z-A^{-1 / 2} A_{0}^{*} A^{1 / 2} z=A y^{*}
$$

It is easy to check that

$$
\left|\left(A^{-1 / 2} A_{0}^{*} A^{1 / 2} z, A z\right)\right| \leq C\left(\left\|y_{e}\right\|_{C(\bar{\Omega})}+\left\|y_{e}\right\|_{C(\bar{\Omega})}\right)\|z\|^{2},
$$

which, together with (3.14), shows that $z \in C([0, T] ; V)$. Hence, $q^{T} \in C([0, T)$; $H)$ as claimed.

By (3.13) and by the unique continuation property for the linear parabolic equation

$$
\begin{gathered}
q_{t}-\left(A+A_{0}\right)^{*} q=0 \quad \text { in } Q, \\
q=0 \quad \text { on } \Gamma,
\end{gathered}
$$

it follows that $q^{T}=q^{T^{\prime}}$ on $(0, T)$ for $0<T<T^{\prime}$. Hence, $q^{T}=q$ is independent of $T$, and so, (3.13) and (3.14) extend to all of $\mathbb{R}^{+}$. Moreover, we have

$$
P y^{0}=-q^{T}(0) \text {. }
$$

Here is the argument. For all $z^{0} \in D\left(A^{1 / 4}\right)$, we have

$$
\begin{aligned}
\varphi\left(y^{0}\right)-\varphi\left(z^{0}\right) \leq \int_{0}^{T}( & \left(A^{3 / 4} y^{*}(t), A^{3 / 4}\left(y^{*}(t)\right.\right. \\
& \left.\left.\left.\quad-z^{*}(t)\right)\right)+\left(u^{*}(t), u^{*}(t)-v^{*}(t)\right)_{\omega}\right) d t \\
& +\left(P y^{*}(T), y^{*}(T)-z^{*}(T)\right),
\end{aligned}
$$

where $\left(z^{*}, v^{*}\right)$ is the optimal pair of problem (3.2) corresponding to $z^{0}$. On the other hand, it follows from (3.14) that

$$
\begin{aligned}
\frac{d}{d t}\left(q^{T}(t), y^{*}(t)-z^{*}(t)\right)= & \left(A^{3 / 4} y^{*}(t), A^{3 / 4}\left(y^{*}(t)-z^{*}(t)\right)\right) \\
& +\left(u^{*}(t), u^{*}(t)-v^{*}(t)\right)_{\omega} .
\end{aligned}
$$

Integrating it on $(0, T)$ and then substituting the result into (3.19), we obtain that

$$
\varphi\left(y^{0}\right)-\varphi\left(z^{0}\right) \leq-\left(q^{T}(0), y^{0}-z^{0}\right),
$$

which yields (3.18) as desired.

By (3.14) and (3.18), we infer that $q(t)=-P y^{*}(t)$ for all $t \geq 0$, which shows (3.8) as desired. 
Now let $y^{0} \in V$. Then, by the previous argument, we see that $q \in C([0, T] ; H)$, which, together with (3.18), shows that $P: V \rightarrow H$. By the closed graph theorem, one obtains (3.9) as desired.

Next we have

$$
\varphi\left(y^{*}(t)\right)=\frac{1}{2} \int_{t}^{\infty}\left(\left|A^{3 / 4} y^{*}(s)\right|^{2}+\left|u^{*}(s)\right|_{\omega}^{2}\right) d s \quad \forall t \geq 0,
$$

and therefore

$$
\left(P y^{*}(t), \frac{d}{d t} y^{*}(t)\right)+\frac{1}{2}\left|A^{3 / 4} y^{*}(t)\right|^{2}+\frac{1}{2}\left|m P y^{*}(t)\right|^{2}=0, \quad \text { a.e. } t>0 .
$$

Since $|m P y| \leq C\|y\|$, for all $y \in V$, we see that the operator $A+A_{0}+m P$ with the domain $D(A)$ generates a $C_{0}$-semigroup in $H$. This implies that $A y^{*}, A_{0} y^{*}$, $m P y^{*} \in C([0, \infty): H)$. Then, it follows from (3.23) that, for $y^{0} \in D(A)$,

$$
\begin{gathered}
-\left(P y^{*}(t), A y^{*}(t)+A_{0} y^{*}(t)\right)-\frac{1}{2}\left|m P y^{*}(t)\right|^{2} \\
+\frac{1}{2}\left|A^{3 / 4} y^{*}(t)\right|^{2}=0 \quad \forall t \geq 0,
\end{gathered}
$$

which implies (3.11) thereby completing the proof.

Proof of Theorem 2.1. Let $P$ be the operator given in Lemma 3.1. Consider the closed-loop system

$$
\begin{gathered}
y_{t}+A y+f(x, y, \nabla y)+m P\left(y-y_{e}\right)=0, \quad t>0, \\
y(0)=y_{0} .
\end{gathered}
$$

It is well known (see [8, Theorem 8.4.5]) that, under hypotheses $\left(\mathrm{H}_{1}\right)$ and $\left(\mathrm{H}_{2}\right)$, (3.25) has a unique local solution $y \in L^{2}(0, T ; V) \cap C([0, T) ; H)$ for each $y_{0} \in$ $W, u \in L^{2}\left(\mathbb{R}^{+} ; H\right)$, where $T>0$. Moreover, $t^{1 / 2} y \in L^{2}(0, T ; D(A)) \cap W^{1,2}([0, T)$; $H)$. We will show that if $y_{0} \in W_{\rho}$ for $\rho$ sufficiently small, then this local solution is global and exponentially stabilizes $y_{e}$. To this end, we substitute $y$ by $y+y_{e}$ into (3.25) and reduce the problem to that of stability of the null solution to equation

$$
\begin{gathered}
y_{t}+A y+A_{0} y+R(y)+m P y=0, \quad t>0, \\
y(0)=y^{0} \equiv y_{0}-y_{e},
\end{gathered}
$$

where $R(y)=f\left(x, y+y_{e}, \nabla\left(y+y_{e}\right)\right)-f\left(x, y_{e}, \nabla y_{e}\right)-A_{0} y$. By hypotheses $\left(\mathrm{H}_{1}\right)$ and $\left(\mathrm{H}_{2}\right)$, we see that

$$
|R(y)| \leq C\left(\sum_{i=1}^{m_{0}}|y|^{p_{i}}+\sum_{j=1}^{l_{0}}|\nabla y|^{q_{j}}\right)
$$


for some positive integers $m_{0}$ and $l_{0}$, where $p_{i}$ and $q_{j}$ satisfy $1<p_{1}<\cdots<p_{m_{0}}$, $1<q_{1}<\cdots<q_{l_{0}}$, and conditions (i), (ii), (iii), and (iv) in hypothesis $\left(\mathrm{H}_{2}\right)$.

We multiply (3.26) by $P y$ and use (3.11) to get, after some calculation, that

$$
\begin{aligned}
& \frac{d}{d t}(P y(t), y(t))+|m P y(t)|^{2}+\left|A^{3 / 4} y(t)\right|^{2} \\
& \quad \leq 2|(P y(t), R(y(t)))|, \quad \text { a.e. } t \in(0, T)
\end{aligned}
$$

We will show that there exist $C>0$ and $\lambda>0$ independent of $y$ such that

$$
|(P y, R(y))| \leq C\left|A^{1 / 4} y\right|^{\lambda}\left|A^{3 / 4} y\right|^{2} \quad \forall y \in D\left(A^{3 / 4}\right) .
$$

From (3.27), it is sufficient to show that

$$
\begin{array}{cc}
\left|\left(P y,|y|^{p}\right)\right| \leq C\left|A^{1 / 4} y\right|^{p-1}\left|A^{3 / 4} y\right|^{2} & \forall y \in D\left(A^{3 / 4}\right), \\
\left|\left(P y,|\nabla y|^{q}\right)\right| \leq C\left|A^{1 / 4} y\right|^{q-1}\left|A^{3 / 4} y\right|^{2} & \forall y \in D\left(A^{3 / 4}\right),
\end{array}
$$

where $p>1$ and $q>1$ satisfy conditions (i), (ii), (iii), and (iv) in hypothesis $\left(\mathrm{H}_{2}\right)$.

We recall that $D\left(A^{s}\right)=H_{0}^{2 s}(\Omega)$ for $s>1 / 4$ and $D\left(A^{1 / 4}\right) \subset H^{1 / 2}(\Omega)$. Thus the norm $|\cdot|_{D\left(A^{s}\right)}$ and $\|\cdot\|_{2 s}$ are equivalent for $s>1 / 4$.

We have, by (3.9) and by interpolation inequality, that

$$
\left|\left(P y,|y|^{p}\right)\right| \leq C\|y\||y|_{2 p}^{p} \leq C\left|A^{1 / 4} y\right|^{1 / 2}\left|A^{3 / 4} y\right|^{1 / 2}|y|_{2 p}^{p},
$$

while, by Sobolev's imbedding theorem,

$$
|y|_{2 p} \leq C\|y\|_{\alpha}
$$

for $\alpha \geq n(1 / 2-1 / 2 p)$. We assume that $1 / 2<\alpha \leq 3 / 2$ and $n>1$, that is,

$$
1<p \leq \frac{n}{n-2 \alpha}
$$

Then again, by the interpolation inequality, we obtain that

$$
\|y\|_{\alpha} \leq C\left|A^{1 / 4} y\right|^{3 / 2-\alpha}\left|A^{3 / 4} y\right|^{\alpha-1 / 2} .
$$

This, together with (3.32), implies that

$$
\left|\left(P y,|y|^{p}\right)\right| \leq C\left|A^{1 / 4} y\right|^{1 / 2+(3 / 2-\alpha) p}\left|A^{3 / 4} y\right|^{1 / 2+(\alpha-1 / 2) p} .
$$

Similarly, taking into account that

$$
|\nabla y|_{2 q} \leq C\|\nabla y\|_{\beta} \quad \text { for } \frac{1}{2 q} \geq \frac{1}{2}-\frac{\beta}{n},
$$


706 Feedback stabilization of semilinear heat equations

we have, by the interpolation inequality, that

$$
\begin{aligned}
\left|\left(P y,|\nabla y|^{q}\right)\right| & \leq|P y||\nabla y|_{2 q}^{q} \leq C\left|A^{1 / 4} y\right|^{1 / 2}\left|A^{3 / 4} y\right|^{1 / 2}\|y\|_{\beta+1} \\
& \leq C\left|A^{1 / 4} y\right|^{1 / 2}\left|A^{3 / 4} y\right|^{1 / 2}\left|A^{1 / 4} y\right|^{(1 / 2-\beta) q}\left|A^{3 / 4} y\right|^{(1 / 2+\beta) q} \\
& \leq C\left|A^{1 / 4} y\right|^{1 / 2+q(1 / 2-\beta)}\left|A^{3 / 4} y\right|^{1 / 2+(1 / 2+\beta) q},
\end{aligned}
$$

where $\beta$ and $q$ are such that

$$
0<\beta \leq \frac{1}{2}, \quad 1<q \leq \frac{n}{n-2 \beta} .
$$

Assume first that $n>1$. Then in virtue of (3.36), inequality (3.30) is satisfied if, besides (3.34), we also have that

$$
\left(\alpha-\frac{1}{2}\right) p \leq \frac{3}{2} \quad \text { for some } \alpha \in\left(\frac{1}{2}, \frac{3}{2}\right]
$$

But it is easily seen that both (3.34) and (3.40) are satisfied if

$$
\begin{array}{ll}
1<p \leq \frac{n+3}{n-1} & \text { for } 1<n \leq 9 \\
1<p \leq \frac{n}{n-3} & \text { for } n>9
\end{array}
$$

Similarly, it follows by (3.38) that estimate (3.31) holds if, besides (3.39), $q$ also satisfies the condition

$$
\frac{1}{2}+\left(\frac{1}{2}+\beta\right) q \leq 2
$$

By an elementary calculation, it follows that (3.39) and (3.42) hold for some $\beta \in(0,1 / 2]$ if

$$
\begin{array}{ll}
1<q \leq \frac{n+3}{n+1} & \text { for } 1 \leq n<3, \\
1<q \leq \frac{n}{n-1} & \text { for } n \geq 3 .
\end{array}
$$

Note that, in this case, $n=1$ is allowed.

If $n, p, q$ satisfy conditions (ii), (iii), (iv) of hypothesis $\left(\mathrm{H}_{2}\right)$, then clearly (3.41) and (3.43) (and consequently (3.30) and (3.31)) are satisfied.

Consider now the case where $n=1$. In this case, $D\left(A^{1 / 4}\right) \subset L^{p}(\Omega)$ for all $p \geq$ 1 , and so, we get, as above, that

$$
\left|\left(P y,|y|^{p}\right)\right| \leq C\left|A^{1 / 4} y\right|^{p+1 / 2}\left|A^{3 / 4} y\right|^{1 / 2}
$$


for all $p$. Inequality (3.31), in this case, follows from (3.43). This completes the proof of (3.30) and (3.31).

Now we come back to (3.28) and set $E=\left\{y^{0} \in W ;\left(P y^{0}, y^{0}\right)<\rho\right\}$. Then, by (3.28) and (3.29), we see that for $\rho$ small enough, if $y^{0} \in E$, we have

$$
\frac{d}{d t}(P y(t), y(t))+\frac{1}{2}\left|A^{3 / 4} y(t)\right|^{2} \leq 0, \quad \text { a.e. } t \in(0, T)
$$

where $(0, T)$ is the maximal interval of existence of solution, from which it follows that the solution $y$ is global. Moreover, by (3.10), it follows that, for some $\gamma>0$, we have

$$
\frac{d}{d t}(P y(t), y(t))+\gamma(P y(t), y(t)) \leq 0, \quad \text { a.e. } t>0,
$$

and this implies that

$$
\begin{gathered}
\int_{0}^{\infty}\left|A^{3 / 4} y(t)\right|^{2} d t \leq 2\left(P y^{0}, y^{0}\right), \\
|y(t)| \leq|y(t)|_{W} \leq C\left|y^{0}\right|_{W} e^{-\gamma t}, \quad \forall t \geq 0 .
\end{gathered}
$$

This completes the proof.

Remark 3.2. It should be mentioned that the feedback controller $u=-m P(y-$ $\left.y_{e}\right)$ is robust with respect to smooth perturbations. More precisely, if $Q_{\varepsilon} \in L(V$, $H)$ is such that $\left|Q_{\varepsilon} y\right| \leq \delta(\varepsilon)|P y|$, for all $y \in V$, where $\delta(\varepsilon) \rightarrow 0$ as $\varepsilon \rightarrow 0$, then for $\varepsilon>0$ sufficiently small, the feedback controller $u=-m\left(P+Q_{\varepsilon}\right)\left(y-y_{e}\right)$ still exponentially stabilizes $y_{e}$ in the neighborhood $W_{\rho}$. Indeed, if we multiply equation

$$
\begin{gathered}
y_{t}+\left(A+A_{0}\right) y+m\left(P+Q_{\varepsilon}\right) y+R(y)=0, \quad t \geq 0, \\
y(0)=y^{0}
\end{gathered}
$$

by $P y$ and proceed as above, we find that

$$
\begin{aligned}
& \frac{d}{d t}(P y, y)+\left|A^{3 / 4} y\right|^{2}+\frac{1}{2}|m P y|^{2} \\
& \leq|(R(y), P y)|+\left|Q_{\varepsilon} y\right||m P y| \\
& \leq|(R(y), P y)|+\frac{1}{2}|m P y|^{2} \\
& \quad+\delta(\varepsilon)|P y|^{2}, \quad \forall t>0,
\end{aligned}
$$

which, in virtue of (3.9) and (3.29), implies the desired result for $\varepsilon$ small enough. This is in particular useful when one replaces an exact solution $P$ to (3.11) by an approximating one. (See also the example which follows.)

We can illustrate the above result by two examples. 
Example 3.3. Consider the controlled equation

$$
\begin{gathered}
y_{t}-\Delta y-\lambda s(x) f(y)=m u, \quad x \in \Omega, t>0, \\
y=0 \quad \text { on } \partial \Omega \times(0, \infty), \\
y(x, 0)=y_{0}(x) \quad \text { in } \Omega,
\end{gathered}
$$

where $\Omega \in \mathbb{R}^{n}, n \leq 3$, is a bounded and open connected subset with $C^{2}$ boundary, $\lambda>0$ is a constant, $s \in L^{\infty}(\Omega)$, and $y_{0} \in H_{0}^{1}(\Omega)$ are given functions such that $0 \leq y_{0}(x) \leq 1$, a.e. $x \in \Omega, f(y)=y(1-y)(a y+(1-a)(1-y))$ for a constant $a$ in $(0,1)$. This equation describes the change of gene frequency in a population to migration and selection (see [4, page 315]). It turns out that if $\lambda>\lambda_{0}$, where $\lambda_{0}$ is the first eigenvalue for the linearized equation, then the zero solution to the steady-state equation is unstable.

If we apply Theorem 2.1 to (3.50), we can conclude that the feedback law $u=-m P y$ locally exponentially stabilizes (3.50), where $P$ is the solution to the algebraic Riccati equation (3.11), that is,

$$
\left(A+A_{0}\right) P+P\left(A+A_{0}\right)+P B B^{*} P=A^{3 / 2},
$$

where $B u=m u$ and $A_{0} y=f^{\prime}(0) s(x) y$.

By the Schwartz-Kernel theorem (see [6, page 157]), we may represent $P$ as

$$
P y(x)=\int_{\Omega} \tilde{P}(x, \xi) y(\xi) d \xi \quad \forall y \in \mathscr{D}(\Omega),
$$

where $\tilde{P}(x, \xi)$ is a distribution on $\Omega_{x} \times \Omega_{\xi}$ determined uniquely by $P$. Then, it follows from (3.51), after some calculation, that $\tilde{P}$ is the solution to the elliptic equation

$$
\begin{gathered}
-\Delta_{x} \tilde{P}(x, \xi)-\Delta_{\xi} \tilde{P}(x, \xi)-\lambda f^{\prime}(0)(s(x)+s(\xi)) \tilde{P}(x, \xi)+\int_{\omega} \tilde{P}(x, \eta) \tilde{P}(\eta, \xi) d \eta \\
=A^{3 / 2} \delta(x-\xi) \quad \text { in } \Omega \times \Omega, \\
\tilde{P}=0 \quad \text { on } \partial \Omega \times \partial \Omega, \quad \tilde{P}(x, \xi)=\tilde{P}(\xi, x) \quad \forall x, \xi \in \Omega,
\end{gathered}
$$

where $A^{3 / 2} \delta(x-\xi)(\varphi)=A^{3 / 2} \varphi(x)$ for all $\varphi \in \mathscr{D}(\Omega)$.

Equation (3.53) can be approximated by

$$
\begin{gathered}
-\Delta_{x} \tilde{P}_{N}(x, \xi)-\Delta_{\xi} \tilde{P}_{N}(x, \xi)+\lambda f^{\prime}(0)(s(x)+s(\xi)) \tilde{P}_{N}(x, \xi) \\
+\int_{\omega} \tilde{P}_{N}(x, \eta) \tilde{P}_{N}(\eta, \xi) d \eta \\
=\sum_{j=1}^{N} \lambda_{j}^{3 / 2} \varphi_{j}(x) \varphi_{j}(\xi), \quad(x, \xi) \in \Omega \times \Omega, \\
\tilde{P}_{N}(x, \xi)=0 \quad \text { on } \partial \Omega \times \partial \Omega,
\end{gathered}
$$


where $N$ is a natural number and $\left(\lambda_{j}, \varphi_{j}\right)$ are the eigenvalues and eigenfunctions, respectively, to the Laplace operator with Dirichlet homogeneous boundary value conditions. According to Remark 3.2, one might suggest that the feedback controller $u=-m P_{N}\left(y-y_{e}\right)$ is a local stabilizing feedback for (3.50).

Example 3.4. The steady-state solution $y_{e}=0$ to nonlinear heat equation

$$
\begin{gathered}
y_{t}-y_{x x}-a y+b y^{3}=m u \quad \text { in }(0, \pi) \times(0, \infty), \\
y(0, t)=y(\pi, t)=0 \quad \text { in }(0, \infty), \\
y(x, 0)=y_{0}(x) \quad \text { in }(0, \pi),
\end{gathered}
$$

is unstable for $a>1$ (see [4]). However, according to Theorem 2.1, the feedback controller $u=-P y$, where $P$ is the solution to the algebraic Riccati equation (3.11).

Equivalently,

$$
\begin{gathered}
-\tilde{P}_{x x}(x, \xi)-\tilde{P}_{\xi \xi}(x, \xi)-2 a \tilde{P}(x, \xi)+\int_{\omega} \tilde{P}(x, \eta) \tilde{P}(\eta, \xi) d \eta=A^{3 / 2} \delta(x-\xi), \\
\tilde{P}(0,0)=\tilde{P}(\pi, \pi)=0
\end{gathered}
$$

exponentially stabilizes (3.55) in a neighborhood of the origin.

\section{Boundary feedback stabilization of semilinear equation}

In this section, we will prove Theorem 2.2. Proceeding as above, we consider the linearized form of (2.12), that is,

$$
z^{\prime}+A z+A_{1} z=B u, \quad t>0, z(0)=z_{0},
$$

and the linear quadratic optimal control problem

$$
\psi\left(z_{0}\right)=\operatorname{Min}\left\{\frac{1}{2} \int_{0}^{\infty}\left(\left|A^{1 / 4-\varepsilon} z(t)\right|^{2}+|u(t)|_{U}^{2}\right) d t, \text { subject to }(4.1)\right\}
$$

where $\varepsilon>0$ is arbitrarily small but fixed. By a similar argument as that used in the proof of Lemma 3.1, taking into account (2.11) and the boundary exact null controllability of linear parabolic equation (see $[1,3]$ ), we obtain the following lemma.

LemmA 4.1. There are the constants $c_{i}>0, i=1,2$, such that

$$
c_{2}\left|A^{-1 / 4-\varepsilon} z_{0}\right|^{2} \leq \psi\left(z_{0}\right) \leq c_{1}\left|A^{-1 / 4-\varepsilon} z_{0}\right|^{2} \quad \forall z_{0} \in\left(D\left(A^{1 / 4+\varepsilon}\right)\right)^{\prime},
$$

where $\left(D\left(A^{1 / 4+\varepsilon}\right)\right)^{\prime}$ denotes the dual of $D\left(A^{1 / 4+\varepsilon}\right)$ in the pairing induced by $H$.

Now we are ready to prove Theorem 2.2. 
710 Feedback stabilization of semilinear heat equations

Proof of Theorem 2.2. Let $P:\left(D\left(A^{1 / 4+\varepsilon}\right)\right)^{\prime} \rightarrow D\left(A^{1 / 4+\varepsilon}\right)$ be the differential of $\psi$, that is,

$$
\psi\left(z_{0}\right)=\frac{1}{2}\left(P z_{0}, z_{0}\right) \quad \forall z_{0} \in\left(D\left(A^{1 / 4+\varepsilon}\right)\right)^{\prime} .
$$

Let $\left(z^{*}, u^{*}\right)$ be optimal for problem (4.2). By a similar argument to the proof of Lemma 3.1, it follows that, for each $T>0$,

$$
\begin{gathered}
u^{*}(t)=B^{*} p(t) \quad \forall t \geq 0, \\
p^{\prime}-A p-A_{1} p=A^{1 / 2-2 \varepsilon} z^{*}, \quad t \in(0, T), \\
p(T)=-P z^{*}(T), \\
P z_{0}=-p(0) .
\end{gathered}
$$

Note that the solution $p$ to (4.6) satisfies that $A^{3 / 4+\varepsilon} p \in L^{2}(0, T ; H)$ for all $T>0$, and, by (4.4), (4.5), and (4.7), we have, via dynamic programming theory, that

$$
\frac{d}{d t} \psi\left(z^{*}(t)\right)=-\frac{1}{2}\left(\left|A^{1 / 4-\varepsilon} z^{*}(t)\right|^{2}+\left|B^{*} P z^{*}(t)\right|_{U}^{2}\right), \quad \text { a.e. } t>0
$$

and therefore

$$
\begin{array}{r}
\left(\left(A+A_{1}\right) z^{*}(t), P z^{*}(t)\right)+\frac{1}{2}\left|B^{*} P z^{*}(t)\right|_{U}^{2} \\
=\frac{1}{2}\left|A^{1 / 4-\varepsilon} z^{*}(t)\right|^{2}, \quad \text { a.e. } t>0 .
\end{array}
$$

On the other hand, it is readily seen, via the dynamic programming principle, that the flow $t \rightarrow z^{*}(t)$ is a $C_{0}$-semigroup on $X \equiv\left(D\left(A^{1 / 4+\varepsilon}\right)\right)^{\prime}$. Let $\mathscr{A}$ be its infinitesimal generator. Then we have

$$
\mathscr{A} z^{*}(t)=A z^{*}(t)+A_{1} z^{*}(t)+B B^{*} P z^{*}(t), \quad \text { a.e. } t>0,
$$

and this implies that

$$
\mathscr{A} z_{0}=\overline{A+A_{1}+B B^{*} P} z_{0} \quad \forall z_{0} \in D(\mathscr{A})
$$

where $\overline{A+A_{1}+B B^{*} P}$ denotes the closure of $A+A_{1}+B B^{*} P$.

We claim that the operator $A+A_{1}+B B^{*} P$ is closed in $X$. Indeed, if $z_{k} \rightarrow z \in X$ as $k \rightarrow \infty$ and $\left(A+A_{1}+B B^{*} P\right) z_{k} \rightarrow \xi$ in $X$, then it follows that

$$
z_{k}+A^{-1} A_{1} z_{k}+A^{-1} B B^{*} P z_{k} \longrightarrow A^{-1} \xi \quad \text { in } D\left(A^{3 / 4-\varepsilon}\right) .
$$

Let $D: U \rightarrow D\left(A^{1 / 4-\varepsilon}\right)$ be defined by $D u=\theta$, where $\theta$ is the solution to (2.10). We have $B=A D$, and so

$$
A^{-1} B B^{*} P z_{k}=D B^{*} P z_{k} .
$$


Let $\theta_{k}=D B^{*} P z_{k}$, then $\theta_{k}$ is the solution to Dirichlet's problem

$$
\Delta \theta_{k}=0 \quad \text { in } \Omega, \quad \theta_{k}=-\frac{\partial}{\partial \gamma}\left(P z_{k}\right) \quad \text { on } \partial \Omega,
$$

because $B^{*} p=-(\partial / \partial \gamma) p$, for all $p \in D(A)$.

It follows, by (4.13), that

$$
\theta_{k}+z_{k}+A^{-1}\left(A_{1} z_{k}\right) \longrightarrow A^{-1} \xi \quad \text { in } D\left(A^{3 / 4-\varepsilon}\right) .
$$

By (4.14), we have

$$
\int_{\Omega} \theta_{k} \Delta \varphi d x=-\int_{\partial \Omega} \frac{\partial \varphi}{\partial \gamma} \frac{\partial}{\partial \gamma}\left(P z_{k}\right) d \sigma \quad \forall \varphi \in H_{0}^{1}(\Omega) \cap H^{2}(\Omega),
$$

while, by (4.15), it follows that

$$
\theta_{k} \longrightarrow \theta \quad \text { in }\left(D\left(A^{1 / 4+\varepsilon}\right)\right)^{\prime} \text { as } k \longrightarrow \infty .
$$

Thus, for $\varphi \in H^{3}(\Omega) \cap H_{0}^{1}(\Omega)$, we have that

$$
\int_{\Omega} \theta \Delta \varphi d x=-\int_{\partial \Omega} \frac{\partial \varphi}{\partial \gamma} \eta d \sigma
$$

where $\eta=\lim _{k \rightarrow \infty}(\partial / \partial \gamma)\left(P z_{k}\right)$ in $H^{-3 / 2}(\partial \Omega)$. Hence, from (4.14) and (4.18), we have that

$$
\Delta \theta=0 \quad \text { in } \Omega, \quad \theta=\eta \quad \text { on } \partial \Omega,
$$

which, together with (4.18), shows that $A^{-1} \xi=D B^{*} P z+z+A^{-1}\left(A_{1} z\right)$ as claimed.

Thus, $\mathscr{A}=A+A_{1}+B B^{*} P$ with the domain $\left\{z \in H ; A z+A_{1} z+B B^{*} P z \in X\right\}$ generates a $C_{0}$-semigroup on $X$. Then it follows from (4.9) that

$$
\begin{aligned}
((A+ & \left.\left.A_{1}\right) z_{0}, P z_{0}\right)+\frac{1}{2}\left|B^{*} P z_{0}\right|_{U}^{2} \\
& =\frac{1}{2}\left|A^{1 / 4-\varepsilon} z_{0}\right|^{2} \quad \forall z_{0} \in D(\mathscr{A}) \cap D\left(A^{1 / 4-\varepsilon}\right) .
\end{aligned}
$$

Next we consider the closed-loop system

$$
z^{\prime}+A z+A_{1} z+g(z)+B B^{*} P z=0, \quad t>0, \quad z(0)=z_{0} .
$$

We note first that, for each $z_{0} \in D\left(A^{1 / 4-\varepsilon}\right)$, (4.21) has a local mild solution $z \in$ $C\left([0, T] ; D\left(A^{1 / 4-\varepsilon}\right)\right)$ for some $T>0$. Indeed, we may rewrite $(4.21)$ as

$$
z(t)+\int_{0}^{t} S(t-s) g(z(s)) d s=S(t) z_{0}, \quad z_{0} \geq 0
$$


712 Feedback stabilization of semilinear heat equations

where $S(t)$ is the semigroup on $X$ generated by $\mathscr{A}$. By (4.3), we have the estimate

$$
\int_{0}^{\infty}\left|A^{1 / 4-\varepsilon} S(t) z_{0}\right|^{2} d t \leq C\left|A^{-1 / 4-\varepsilon} z_{0}\right|^{2}
$$

On the other hand, we have (via Sobolev's imbedding theorem)

$$
\begin{gathered}
\left|A^{-1 / 4-\varepsilon} g(z)\right| \leq \sup \left\{|(g(z), \varphi)| ;\left|A^{1 / 4+\varepsilon} \varphi\right| \leq 1\right\}, \\
C \leq \sup \left\{|(g(z), \varphi)| ;|\varphi|_{L^{p^{*}}(\Omega)} \leq 1\right\} \leq C|g(z)|_{L^{q^{*}}(\Omega)},
\end{gathered}
$$

where $p^{*}=\infty, q^{*}=1$ if $n=1$, and

$$
p^{*}=\frac{2 n}{n-(1+4 \varepsilon)}, \quad q^{*}=\frac{2 n}{n+1+4 \varepsilon} \quad \text { if } n>1
$$

This, together with condition (2.2) in hypothesis $\left(\mathrm{H}_{3}\right)$, implies that

$$
\left|A^{-1 / 4-\varepsilon} g(z)\right| \leq C\left(\int_{\Omega}|z|^{p q^{*}} d x\right)^{1 / q^{*}},
$$

which, combined with Sobolev's imbedding theorem, shows that, for $p \leq(n+$ $1+4 \varepsilon) /(n-1+4 \varepsilon)$, we have

$$
\left|A^{-1 / 4-\varepsilon} g(z)\right| \leq C\left|A^{1 / 4-\varepsilon} z\right|^{p} \quad \forall z \in D\left(A^{1 / 4-\varepsilon}\right) .
$$

Denote by $\Psi: C\left([0, T] ; D\left(A^{1 / 4-\varepsilon}\right)\right) \rightarrow C\left([0, T] ; D\left(A^{1 / 4-\varepsilon}\right)\right)$ the integral operator

$$
\Psi(z)(t)=\int_{0}^{t} S(t-s) f(z(s)) d s+S(t) z_{0}
$$

where $T>0$ is arbitrary but fixed. By (4.23) and (4.27), we obtain that

$$
\begin{aligned}
|\Psi(z)(t)|_{D\left(A^{1 / 4-\varepsilon}\right)} & \leq C \int_{0}^{t}|G(t-s)|\left|A^{-1 / 4-\varepsilon} g(z(s))\right| d s+\left|A^{1 / 4-\varepsilon} S(t) z_{0}\right| \\
& \leq C \int_{0}^{t}|G(t-s)|\left|A^{1 / 4-\varepsilon} z(s)\right|^{p} d s+\left|A^{1 / 4-\varepsilon} z_{0}\right|,
\end{aligned}
$$

where $G(t-s)=A^{1 / 4-\varepsilon} S(t-s) A^{1 / 4+\varepsilon}$.

Let $K=\left\{z \in C\left([0, T] ; D\left(A^{1 / 4-\varepsilon}\right)\right) ; \sup _{0 \leq t \leq T}\left|A^{1 / 4-\varepsilon} z(t)\right| \leq M\right\}$. Then $\Psi$ maps $K$ into itself if $T$ is small enough and $M>0$ is large enough. It is also clear that $K$ is compact in $C([0, T] ; H)$ and this implies, by standard Schauder fixed-point theorem, the local existence as claimed.

Now, for $z_{0} \in D\left(A^{1 / 4-\varepsilon}\right) \cap V_{\rho}$, we assume first that the local mild solution $z$ to (4.21) is a strong solution. Then we may multiply (4.21) by $\mathrm{P} z$ and use (4.20) 
and (4.27) to get that

$$
\begin{aligned}
\frac{d}{d t}(P z, z)+\left|A^{1 / 4-\varepsilon} z\right|^{2} & \leq C\left|A^{1 / 4-\varepsilon} z\right|^{p}\left|A^{1 / 4+\varepsilon} P z\right| \\
& \leq C\left|A^{1 / 4-\varepsilon} z\right|^{2}\left|A^{-1 / 4-\varepsilon} z\right|^{p-1}
\end{aligned}
$$

because $P:\left(D\left(A^{1 / 4+\varepsilon}\right)\right)^{\prime} \rightarrow D\left(A^{1 / 4+\varepsilon}\right)$ is continuous and $1<p \leq 2$. This implies that, for $\rho>0$ small enough, we have

$$
\frac{d}{d t}(P z, z)+\frac{1}{2}\left|A^{1 / 4-\varepsilon} z\right|^{2} \leq 0, \quad \text { a.e. } t>0,
$$

which shows that the solution $z$ is global and satisfies estimates (2.14).

If $z$ is not a strong solution, we may approximate (4.21) by

$$
z_{t}+\mathscr{A} z+(I+\lambda \mathscr{A})^{-1} g(z)=0, \quad t>0, \quad z(0)=z_{0}
$$

where $\lambda>0$. By the same argument as above, (4.32) has a local mild solution $z_{\lambda}$ which must be a strong solution because $(I+\lambda \mathscr{A})^{-1} g: D\left(A^{1 / 4-\varepsilon}\right) \rightarrow X$ is continuously differentiable. Then we may get estimate (4.31) for each $z_{\lambda}, \lambda>0$. Passing to the limit for $\lambda \rightarrow 0$, we obtain (4.31) for a local mild solution $z$ to (4.21). This completes the proof.

\section{Acknowledgments}

This work was done during the visit of the first author (V. Barbu) to The Center for Optimal Control and Discrete Mathematics of Huazhong Normal University of China. The author is grateful to this center for warm hospitality. This work was supported by the Excellent Youth Teacher Foundation of the Ministry of National Education of China and National Natural Science Foundation of China, Grant 0071028 .

\section{References}

[1] V. Barbu, Controllability of parabolic and Navier-Stokes equations, Sci. Math. Jpn. 56 (2002), no. 1, 143-211.

[2] - Feedback stabilization of Navier-Stokes equations, ESAIM COCV 9 (2003), 197-206.

[3] A. V. Fursikov and O. Yu. Imanuvilov, Controllability of Evolution Equations, Lecture Notes Series, vol. 34, Seoul National University, Seoul, 1996.

[4] D. Henry, Geometric Theory of Semilinear Parabolic Equations, Lecture Notes in Mathematics, vol. 840, Springer-Verlag, Berlin, 1981.

[5] I. Lasiecka and R. Triggiani, Control Theory for Partial Differential Equations: Continuous and Approximation Theories. I, Encyclopedia of Mathematics and Its Applications, vol. 74, Cambridge University Press, Cambridge, 2000.

[6] J.-L. Lions, Optimal Control of Systems Governed by Partial Differential Equations, Die Grundlehren der mathematischen Wissenschaften, vol. 170, Springer-Verlag, New York, 1971. 


\section{Feedback stabilization of semilinear heat equations}

[7] J.-L. Lions and E. Magenes, Non-Homogeneous Boundary Value Problems and Applications. Vol. I, Die Grundlehren der mathematischen Wissenschaften, vol. 181, Springer-Verlag, New York, 1972.

[8] A. Pazy, Semigroups of Linear Operators and Applications to Partial Differential Equations, Applied Mathematical Sciences, vol. 44, Springer-Verlag, New York, 1983.

V. Barbu: Department of Mathematics, "Alexandru Ioan Cuza" University, 6600 Iasi, Romania

E-mail address: barbu@uaic.ro

G. Wang: Department of Mathematics and The Center for Optimal Control and Discrete Mathematics, Huazhong Normal University, Wuhan 430079, China

E-mail address: wanggs@ccnu.edu.cn 


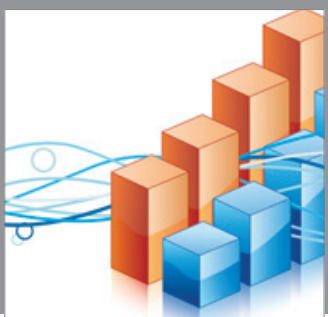

Advances in

Operations Research

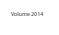

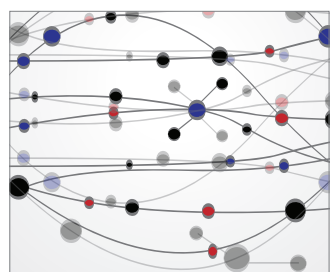

\section{The Scientific} World Journal
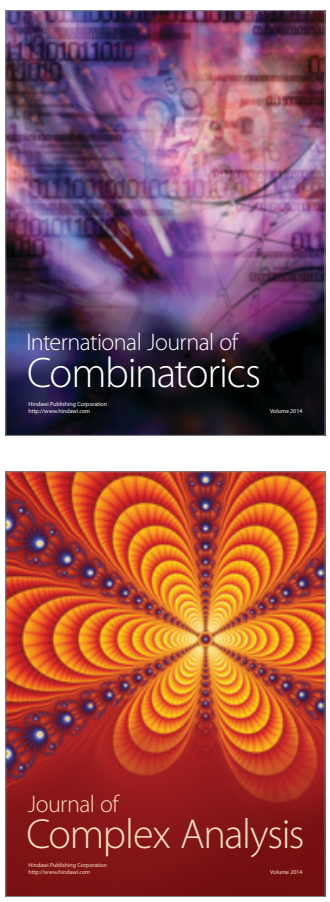

International Journal of

Mathematics and

Mathematical

Sciences
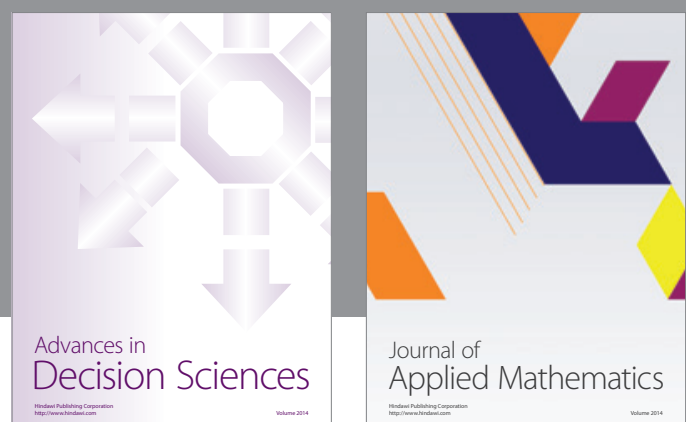

Journal of

Applied Mathematics
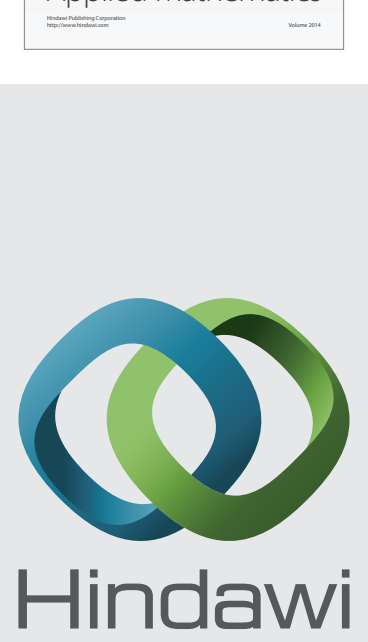

Submit your manuscripts at http://www.hindawi.com
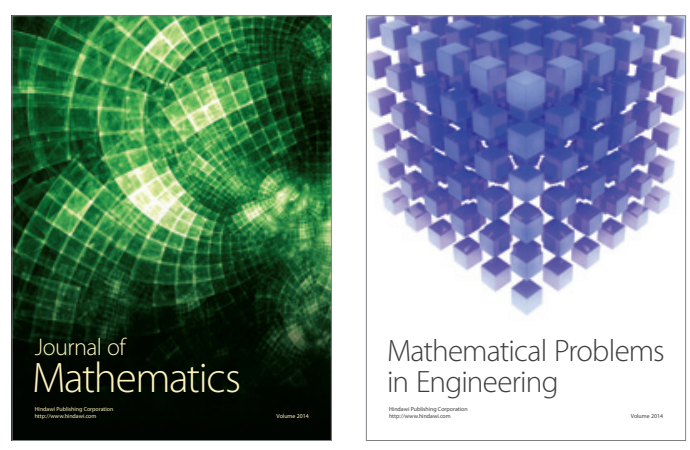

Mathematical Problems in Engineering
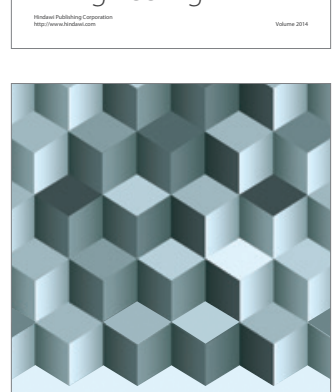

Journal of

Function Spaces
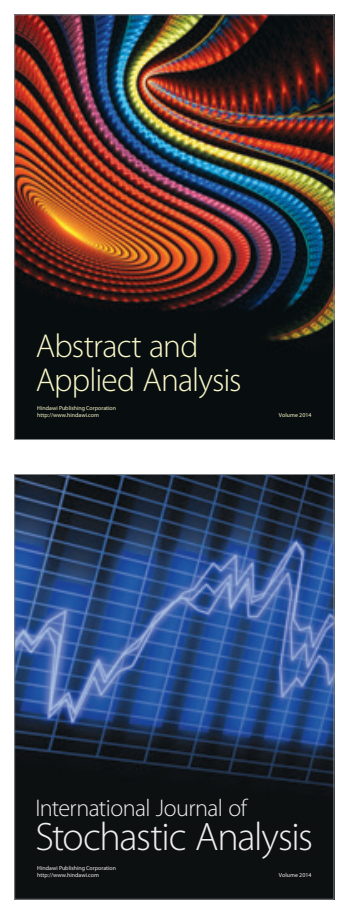

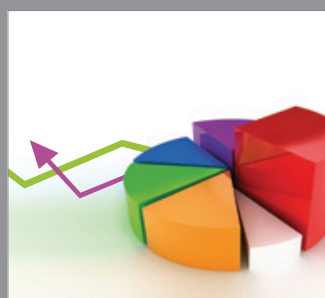

ournal of

Probability and Statistics

Promensencen
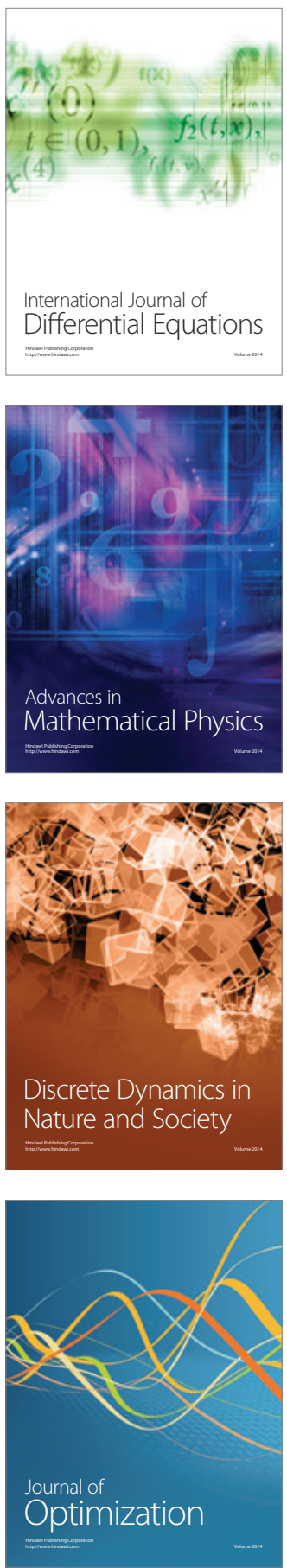